\title{
Resist materials for 157-nm microlithography: an update
}

Raymond Jui-Pu Hung, Hoang Vi Tran, Brian C. Trinque, Takashi Chiba, Shintaro Yamada, et al.

Raymond Jui-Pu Hung, Hoang Vi Tran, Brian C. Trinque, Takashi Chiba, Shintaro Yamada, Daniel Sanders, Eric F Connor, Robert H. Grubbs, John M. Klopp, Jean M. J. Frechet, Brian H. Thomas, Gregory John Shafer, Darryl D DesMarteau, Will Conley, C. Grant Willson, "Resist materials for 157-nm microlithography: an update," Proc. SPIE 4345, Advances in Resist

Technology and Processing XVIII, (24 August 2001); doi: 10.1117/12.436870 Clara, CA, United States 


\title{
Resist Materials for $157 \mathrm{~nm}$ Microlithography: An Update
}

\author{
Raymond J. Hung ${ }^{\mathrm{a}}$, Hoang V. Tran ${ }^{\mathrm{a}}$, Brian C. Trinque ${ }^{\mathrm{a}}$, Takashi Chiba ${ }^{\mathrm{a}}$, Shintaro Yamada ${ }^{\mathrm{a}, \mathrm{f}}$, \\ Daniel P. Sanders ${ }^{b}$, Eric F. Connor ${ }^{b}$, Robert H. Grubbs ${ }^{b}$, John Klopp ${ }^{c}$, Jean M. J. Frechet, \\ Brian H. Thomas ${ }^{\mathrm{d}}$, Gregory J. Shafer ${ }^{\mathrm{d}}$, Darryl D. DesMarteau ${ }^{\mathrm{d}}$, Will Conley ${ }^{\mathrm{e}}$, and C. Grant Willson ${ }^{\mathrm{a}}$ \\ ${ }^{a}$ Departments of Chemistry and Chemical Engineering, University of Texas, Austin, TX 78712 \\ ${ }^{b}$ Department of Chemistry, California Institute of Technology, Pasadena, CA 91125 \\ ${ }^{c}$ Department of Chemistry, University of California, Berkeley, CA 94720 \\ ${ }^{d}$ Department of Chemistry, Clemson University, Clemson, SC, 29634 \\ ${ }^{e}$ International SEMATECH, 2706 Montopolis Drive, Austin, TX 78741-6499 \\ ${ }^{f}$ Current Address: Shipley, 455 Forest Street, Marlboro, MA 01752
}

\begin{abstract}
Fluorocarbon polymers and siloxane-based polymers have been identified as promising resist candidates for $157 \mathrm{~nm}$ material design because of their relatively high transparency at this wavelength. ${ }^{1}$ This paper reports our recent progress toward developing $157 \mathrm{~nm}$ resist materials based on the first of these two polymer systems. In addition to the 2hydroxyhexafluoropropyl group, $\alpha$-trifluoromethyl carboxylic acids have been identified as surprisingly transparent acidic functional groups. Polymers based on these groups have been prepared and preliminary imaging studies at $157 \mathrm{~nm}$ are described. 2-(Trifluoromethyl)bicyclo[2,2,1]heptane-2-carboxylic acid methyl ester derived from methyl 2(trifluoromethyl)acrylate was also prepared and gas-phase VUV measurements showed substantially improved transparency over norbornane. This appears to be a general characteristic of norbornane-bearing geminal electron-withdrawing substituents on the 2 carbon bridge. Unfortunately, neither the $\mathrm{Ni}^{\mathrm{II}}$ nor $\mathrm{Pd}^{\mathrm{II}}$ catalysts polymerize these transparent norbornene monomers by vinyl addition. However, several new approaches to incorporating these transparent monomers into functional polymers have been investigated. The first involved the synthesis of tricyclononene (TCN) monomers that move the bulky electron-withdrawing groups further away from the site of addition. The hydrogenated geminally substituted TCN monomer still has far better transparency at $157 \mathrm{~nm}$ than norbornane. The second approach involved copolymerizing the norbornene monomers with carbon monoxide. The third approach involved free-radical polymerization of norbornene monomers with tetrafluoroethylene and/or other electron-deficient comonomers. All these approaches provided new materials with encouraging absorbance at $157 \mathrm{~nm}$. The lithographic performance of some of these polymers is discussed.
\end{abstract}

Keywords: $157 \mathrm{~nm}$ lithography, $157 \mathrm{~nm}$ resist, 2-(trifluoromethyl)acrylates, fluoronorbornanes, metal-catalyzed addition polymerization, carbon monoxide copolymerization, dissolution inhibitor

\section{INTRODUCTION}

The design of $157 \mathrm{~nm}$ photoresists is a daunting task since air, water, and most organic compounds are opaque at this wavelength. Spectroscopic studies ${ }^{1}$ led to the observation that fluorinated hydrocarbons and siloxanes offer the best hope for the transparency that is necessary for the design of an effective $157 \mathrm{~nm}$ photoresist, and these classes of materials have quickly become the prominent platforms for a variety of research activities in this field. Our approach to the design of the resist polymer requires identification of four "modules": a backbone that tethers the functional substituents and provides basic mechanical properties, an etch barrier that provides RIE resistance, an acidic group that generates tetramethylammonium hydroxide (TMAH) developer solubility, and an acid-labile protecting group that enables a chemical amplification type solubility switch. Here, the difficulty lies in identifying moieties that will not only perform these specific functions, but also provide transparency at the $157 \mathrm{~nm}$ wavelength. Our group identified the bistrifluoromethylcarbinol (1,1,1,3,3,3-hexafluoro-2-hydroxypropyl) (HFP) group as a transparent acidic module, and acetal protecting groups were shown to function effectively in conjunction with this structure in an acid-catalyzed, chemically-amplified system (Figure 1). ${ }^{2}$ 
<smiles>CC(C)(C(F)(F)F)C(F)(F)F</smiles><smiles>CCOCOC(C)(C)C(F)(F)F</smiles>

Acidic Group

Figure 1. Transparent acidic module

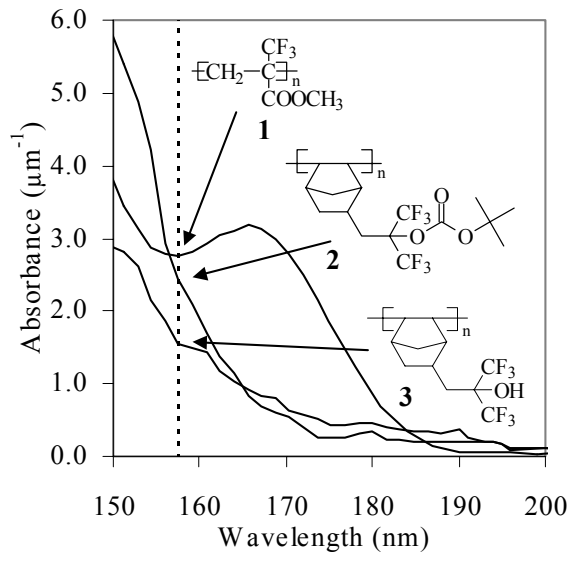

Figure 2. Absorbance of tluorinated polymers

Continuing with this "modular" approach, we reported the relatively low absorbance of poly(methyl 2-(trifluoromethyl)acrylate), which inspired the synthesis of a variety of fluorine-substituted poly(norbornane)s ${ }^{3 a}$ (Figure 2). Our group has thereby identified polymers that are relatively transparent and fulfill the requirements of the backbone and etch barrier module functions of a $157 \mathrm{~nm}$ resist.

In this paper, we present our continuing synthetic efforts in the area of fluorocarbon-based $157 \mathrm{~nm}$ resist development. We have synthesized a number of fluorinated monomers and have incorporated these into polymer systems by a variety of methods including metal-catalyzed addition polymerizations, carbon monoxide copolymerizations, and anionic and free radical-initiated polymerizations. Investigation of these materials by VUV spectrometry and variable angle spectroscopic ellipsometry (VASE), along with actual imaging experiments of certain polymers, has shown encouraging results and provided a clear direction for the development of $157 \mathrm{~nm}$ lithographic resist materials.

\section{EXPERIMENTAL}

\section{Section 2.1 Materials}

All starting materials were purchased from Aldrich and these were used as received unless noted otherwise. 1,1,1-Trifluoro2-(trifluoromethyl)pent-4-en-2-ol was received as a gift from AZ Clariant and 2-(trifluoromethyl)acrylic acid was received as a gift from Central Glass Co. All solvents were dried by fractional distillation either over $\mathrm{Na} / \mathrm{K}$ metal or $\mathrm{CaH}_{2}$. All liquid reagents used for VUV measurements were distilled from appropriate drying agents, thoroughly degassed by freeze-pumpthaw cycles, and sealed in glass ampoules under vacuum.

\section{Section 2.2 Vacuum UV Spectroscopy}

Gas-phase VUV measurements were made on an Acton CAMS-507 spectrophotometer fitted with a custom-made gas cell attachment. The details of the cell design and implementation are the subject of a separate paper. ${ }^{4}$ VUV spectra of polymer films were calculated from measurements made with a J.A. Woollam VU301 variable angle spectroscopic ellipsometer and/or measured with the Acton CAMS-507 spectrophotometer. The films were cast on calcium fluoride disks from solutions in propylene glycol monomethyl ether acetate (PGMEA) or cyclohexanone and baked at $110^{\circ} \mathrm{C}$ for at least 5 minutes prior to analysis. All absorbance data reported are in base 10.

\section{Section 2.3 Synthesis}

The polyfluorinated acrylate terpolymer (4) was prepared by anionic polymerization ${ }^{3}$ followed by hydrogenolysis of the benzyl ester to provide the free carboxylic acid. The 7,7-difluoronorbornane (6) was made by a 5-step synthesis that will be reported elsewhere. The synthesis of the other fluorinated norbornanes $(\mathbf{5}, \mathbf{7})$ was reported in a previous article. $^{3 \mathrm{a}}$ Bicyclo[2.2.1]hept-5-ene-2-carboxylic acid tert-butyl ester (NBTBE, 8) and bicyclo[2.2.1]hept-5-ene-2-(1,1,1-trifluoro-2trifluoromethylpropan-2-ol (NBHFA, 9) were polymerized by a variety of methods to give poly(NBTBE-co-NBHFA) copolymers (10). 2-(Trifluoromethyl)bicyclo[2.2.1]hept-5-ene-2-carboxylic acid tert-butyl ester $\left(\mathrm{NBCF}_{3} \mathrm{TBE}, \mathbf{1 1}\right)$ was 
prepared by the cycloaddition of the corresponding $t$-butyl ester and cyclopentadiene. These polymers were initiated with (a) $\left(\eta^{6}\right.$-toluene)bis(pentafluorophenyl) nickel(II) in toluene, (b) allyl palladium catalyst in dichloromethane without proton sponges, or (c) allyl palladium catalyst in dichloromethane with proton sponges. ${ }^{6}$ 4,4-Difluoro-3(trifluoromethyl)tricyclo[4.2.1. $0^{2,5}$ ]non-7-ene-3-carboxylic acid methyl ester ( $\mathrm{TCNCF}_{3} \mathrm{ME}, \mathbf{1 3 a}$ ) was synthesized from the cycloaddition of quadricyclane $(\mathbf{1 3})^{5}$ with methyl perfluoromethacrylate ${ }^{13}$. Hydrogenation with $\mathrm{Pd} / \mathrm{C}$ provided the reduced analogue 14 in high yield. Poly $\left(\mathrm{TCNCF}_{3} \mathrm{ME}\right)(\mathbf{1 5})$ was initiated with an allyl palladium catalyst ${ }^{6}$ in dichloromethane at room temperature. The carbon monoxide copolymers $(\mathbf{1 6}, \mathbf{1 7}, \mathbf{1 9}, \mathbf{2 0 a}, \mathbf{2 6})$ were synthesized with Drent's catalyst ${ }^{7}$ in methanol in a sealed Parr reactor at $80^{\circ} \mathrm{C}$ overnight. Polymer 16 was reacted with di-t-butyldicarbonate and 4-dimethylaminopyridine to yield polymer 23. The tetrafluoroethylene copolymers $(\mathbf{2 1}, \mathbf{2 2})$ were prepared at $90^{\circ} \mathrm{C}$ in Freon $113(1,1,2-$ trichlorotrifluoroethane) with AIBN as the initiator. The reactions were run overnight.

\section{Section 2.4 Imaging Conditions}

The following were typical imaging conditions, unless noted otherwise. Wafers were coated with $150 \mathrm{~nm}$ of resist over 82 $\mathrm{nm}$ of bottom antireflective layer and softbaked at $140^{\circ} \mathrm{C}$ for $60 \mathrm{sec}$. Imaging was performed using an alternating phase shift mask on an EXITECH $157 \mathrm{~nm}$ Microstepper at SEMATECH using a numerical aperature of 0.6 and a partial coherence of 0.3. Wafers were then post-exposure baked at $130^{\circ} \mathrm{C}$ for $90 \mathrm{sec}$ and puddle-developed for $20 \mathrm{sec}$ in $2.38 \%$ TMAH. Top down scanning electron micrographs were collected with a JEOL 7550 and cross-sectional data were collected on a Hitachi 4500 scanning electron microscope.

\section{RESULTS AND DISCUSSION}

\section{Section 3.1 Fluorinated Acrylate Platform}

The relatively low absorbances of the alkyl 2-(trifluoromethyl)acrylates prompted the synthesis of a fluorinated acrylate polymer (4) similar to IBM's Version 1193 nm resist. ${ }^{8}$ As shown in Figure 3, the absorbance of this polymer at $157 \mathrm{~nm}$ is approximately $3 \mu \mathrm{m}^{-1}$, which is several orders of magnitude lower than that of the commercially available $248 \mathrm{~nm}$ resist, UV6. However, preliminary imaging experiments using $157 \mathrm{~nm}$ radiation (Figure 4), followed by SEM measurements, indicated that this fluorinated polymer backbone is not stable to e-beam radiation. Some of the polymer vaporized when exposed to high energy electrons from the scanning electron microscope (SEM). This result is not so surprising since this fluorinated acrylate platform was originally designed as an e-beam resist and is clearly very sensitive to main chain scission. ${ }^{9}$ This result led to the decision to explore incorporation of the fluorinated acrylates into polymer structures indirectly rather than directly. One method for doing this is to react the acrylates with cyclopentadiene to give norbornene analogues that can then be polymerized via vinyl addition using an appropriate metal catalyst.

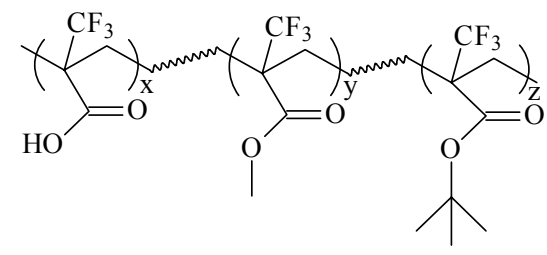

Polymer 4, $x=20, y=40, z=40$

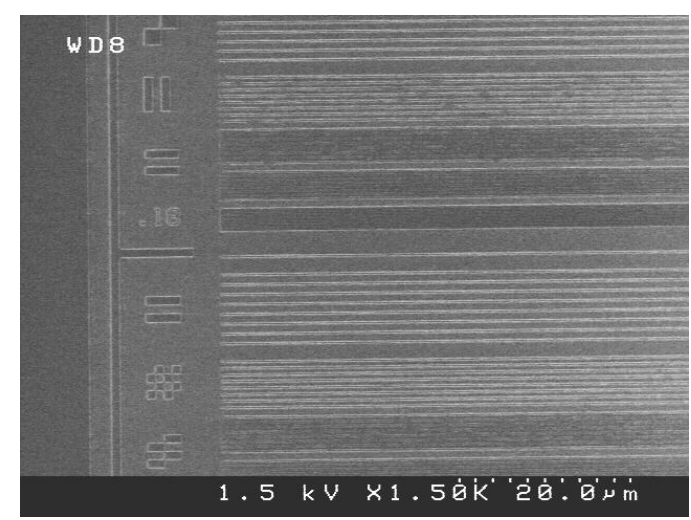

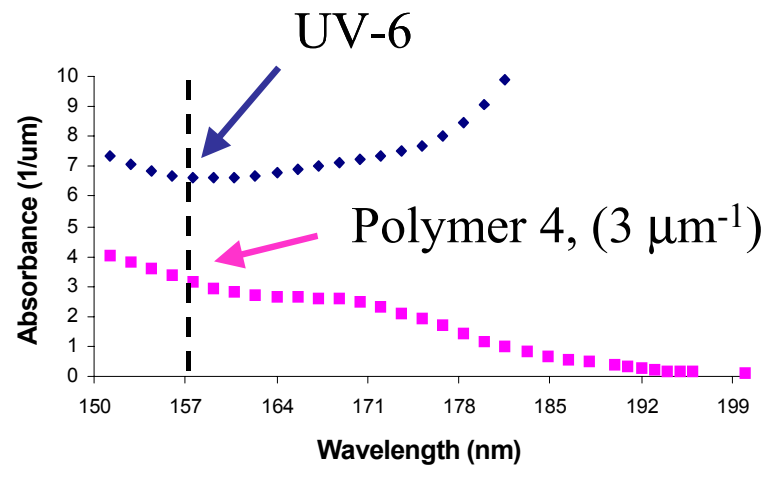

Figure 3. Absorbance of fluorinated acrylate (Polymer 4)

Figure 4. SEM of Pt coated sample of Polymer 4

94.9\% Polymer, $5 \%$ TPS-Nf, $0.1 \% \mathrm{Bu}_{4} \mathrm{NOH}$

$157 \mathrm{~nm}$ exposure (0.6NA-0.7 $)$ ), binary mask, $190 \mathrm{~nm}$ thick on $70 \mathrm{~nm}$ of DUV $30,130^{\circ} \mathrm{C}-60 \mathrm{~s}$ PAB, $130^{\circ} \mathrm{C}-90 \mathrm{~s}$ PEB, 60 sec development $(0.05 \mathrm{~N}$ TMAH $)$ 


\section{Section 3.2 Absorbances of Fluorinated Norbornanes}

The norbornene analogues derived from 2-(trifluoromethyl)acrylates will surely be more absorbing at $157 \mathrm{~nm}$ than the acrylates alone, but offer a trade-off between absorbance and stability. Our group has studied different fluorine substitution patterns on norbornane and how the absorbance of the ring system is influenced by substitution patterns. ${ }^{3}$ Absorbance at 157 $\mathrm{nm}$ is decreased dramatically by selectively fluorinating the norbornane skeleton at key positions (Figure 5). These studies showed that introduction of geminal fluorosubstituents at the two-carbon bridge (5), rather than the one-carbon bridge (6) is the most effective substitution pattern. We therefore reasoned that the introduction of geminal electron-withdrawing groups such as a trifluoromethyl or carboxylalkyl at the 2 position of norbornane should also improve absorbance relative to the parent hydrocarbon.

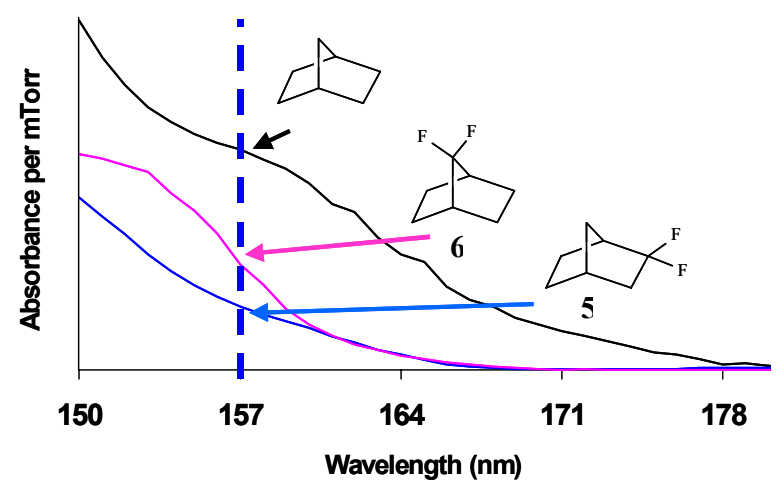

Figure 5. Absorbances of fluorinated norbornanes

Several 2,2-disubstituted norbornanes were therefore prepared by the Diels-Alder addition of cyclopentadiene to some 2(trifluoromethyl)acrylates, and the products were reduced with hydrogen and $\mathrm{Pd} / \mathrm{C}$ to give the corresponding substituted norbornanes (Figure 6). The absorbance of one of these norbornanes, the methyl ester (7), is compared to the absorbance of norbornane and 2,2-difluoronorbornane in Figure 7. This 2-trifluoromethyl-substituted compound is more absorbing at 157 $\mathrm{nm}$ than 2,2-difluoronorbornane, most likely due to carbonyl absorption, but it is far more transparent than norbornane.

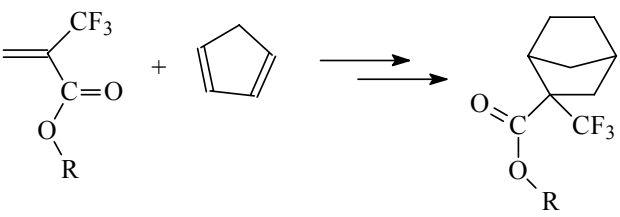

Figure 6. Synthesis of fluorinated 2,2-disubstituted norbornanes

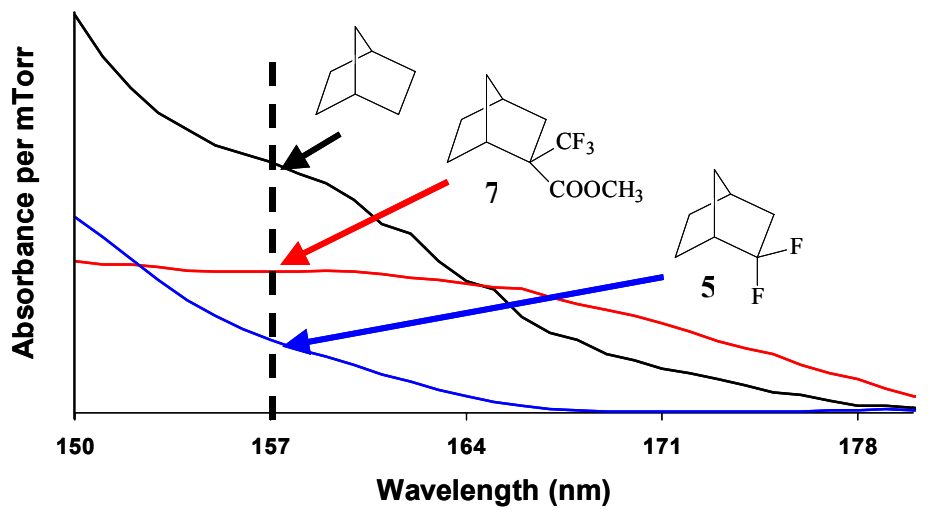

Figure 7. Absorbance of fluorinated norbornane methyl ester

\section{Section 3.3 Metal-Catalyzed Addition Polymerization Studies}

We have previously reported the syntheses and absorbances of a polynorbornene substituted with a HFP group and its $t$-BOC protected derivative (Figure 2). ${ }^{3}$ These polymers, to our surprise, are less absorbing than poly(methyl 2(trifluoromethyl)acrylate). This discovery led us to design resist platforms based on vinyl addition copolymers that incorporate this monomer. The copolymers (10) of NBHFA (9) and NBTBE (8) were successfully made using $\left(\eta^{6}\right.$ toluene)bis(pentafluorophenyl) nickel (II) as the vinyl addition catalyst (Figure 8). The absorbance of one of the co- 
polymers $(\mathrm{x}=80, \mathrm{y}=20)$ is approximately $2.3 \mu \mathrm{m}^{-1}$ at $157 \mathrm{~nm}$. Preliminary imaging results using this co-polymer showed that although the material provides good film coatings, wets well during development and has a high contrast, it suffers from swelling during development. We believe that the high molecular weight of the polymers produced by the nickel catalyst is a major contributor to swelling, and efforts are underway to lower the molecular weight of polymers produced by this catalyst.

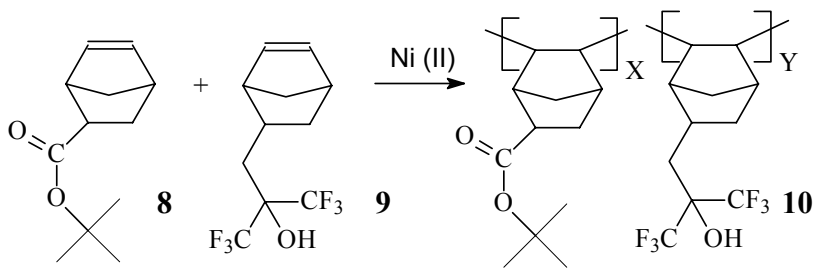

Figure 8. Synthesis of poly(NBHFA-co-NBTBE)

All efforts to incorporate the 2-trifluoromethyl-substituted norbornene esters into a polymer by vinyl addition using the nickel catalyst were unsuccessful. For example, $\mathrm{NBCF}_{3} \mathrm{TBE}(\mathbf{1 1})$ was only incorporated by an amount less than half the feed ratio, when copolymerized with NBHFA (9) (Figure 9). Polymer yields from this reaction are also extremely low $(<10 \%)$. $\mathrm{NBCF}_{3} \mathrm{TBE}$ also did not copolymerize with NBHFA with an allyl palladium catalyst prepared using a literature procedure. ${ }^{6}$

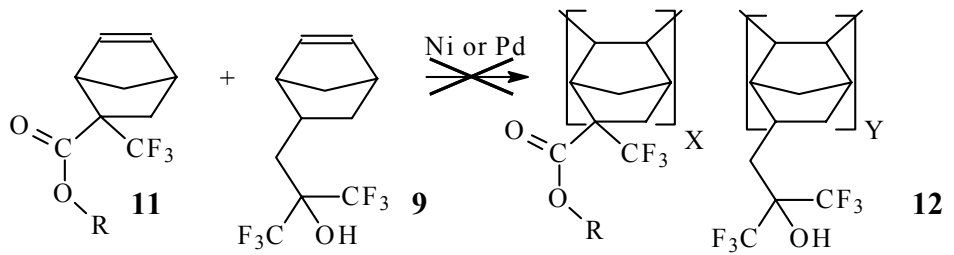

Figure 9. Attempted synthesis of poly(NBHFA-co-NBCF 3 TBE, $\mathrm{R}=t$-butyl, $\mathrm{x}=14, \mathrm{y}=86$

The allyl palladium catalyst does copolymerize NBHFA and NBTBE to yield polymer 10, but the acid-labile functional groups can be deprotected in reactions based on this allyl palladium catalyst. The formation of carboxylic acids is observed from attempts to polymerize NBTBE using this catalyst. The source of the strong acid that causes this deprotection may be reductive elimination of palladium hydride that forms during the polymerization. Whatever the source of this acid, the best solution is to quench it using sterically hindered, polymer-bound pyridines or "proton sponges". These hindered amines can neutralize any residual acid without significantly affecting the active catalyst (by irreversible binding to the active catalyst). The other problem with this polymerization is that it generates product with very high molecular weight. The use of typical chain transfer agents on the polymerization of monomer 9 was unsuccessful (Figure 10). Addition of 1-hexene did not reduce the molecular weight of the polymer to an acceptable degree. Another, more expensive, solution is to simply use a higher catalyst loading. This method worked well with the palladium catalyst (Figure 11), but did not work with the nickel catalyst. In summary, we successfully solved both of the problems that plagued the allyl palladium catalyzed addition polymerization by using proton sponges and higher catalyst loadings. The use of large amounts of expensive catalyst is not a satisfying solution but it is a workable one, and provides resist material while we search for an effective chain transfer agent.

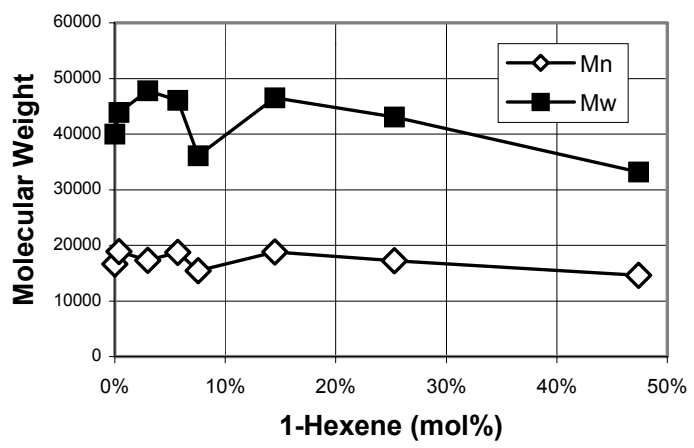

Figure 10. Effect of 1-hexene as a chain transfer agent on the molecular weight of polymer 3 (from monomer 9)

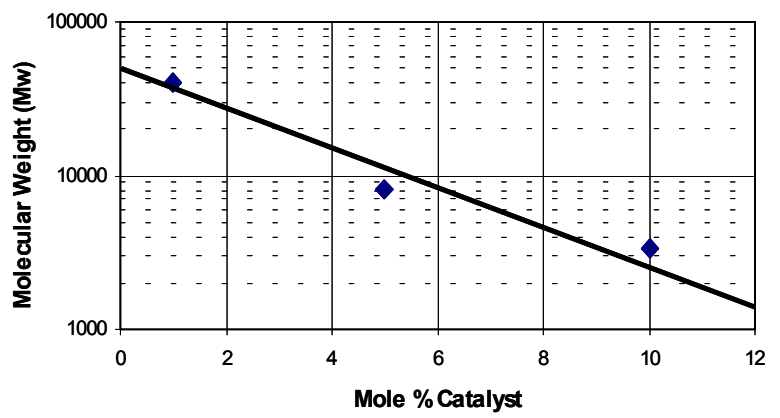

Figure 11. Effect of added Pd catalyst on molecular weight of polymer $\mathbf{3}$ (from monomer 9) 


\section{Section 3.4 Incorporation of Geminal Substituted Monomers}

Incorporation of geminal substituted monomers such as $\mathrm{NBCF}_{3} \mathrm{TBE}$ into a polymer did not work well using either nickel (II) or palladium (II) catalysts. The reason for this may stem from the reduced electron density of the double bond resulting from the close proximity of the electron-withdrawing groups, from steric inhibition, or both. It should be noted that these vinyl addition catalysts typically polymerize electron-rich olefins much faster than electron-poor olefins. ${ }^{6}$ We have explored several approaches to incorporating these monomers into a polymer system. The first was to move the trifluoromethyl group further away from the double bond by synthesizing the corresponding tricyclononene (TCN) analogues. The second method involved copolymerization of carbon monoxide with the trifluoromethyl-substituted norbornene monomers using a different catalyst system. The third method was to use free-radical mediated polymerization with electron deficient monomers, for example, tetrafluoroethylene (TFE) to make alternating copolymers.

\section{Section 3.4.1 Tricyclononene (TCN) Monomers}

We have successfully synthesized the TCN model compound 14 (Figure 12) and compared its absorbance to that of norbornane and 2,2-difluoronorbornane (Figure 13). Compound 14 shows improved transparency over the norbornane hydrocarbon, so a variety of TCN derivatives are currently being prepared. The $\mathrm{TCNCF}_{3} \mathrm{ME}$ monomer was successfully polymerized by the allyl palladium catalyst described earlier (Figure 14), and its absorbance at $157 \mathrm{~nm}$ was found to be 2.8 $\mu \mathrm{m}^{-1}$. Therefore, the TCN system has significantly improved reactivity towards vinyl addition polymerization while retaining a remarkable absorbance at $157 \mathrm{~nm}$.

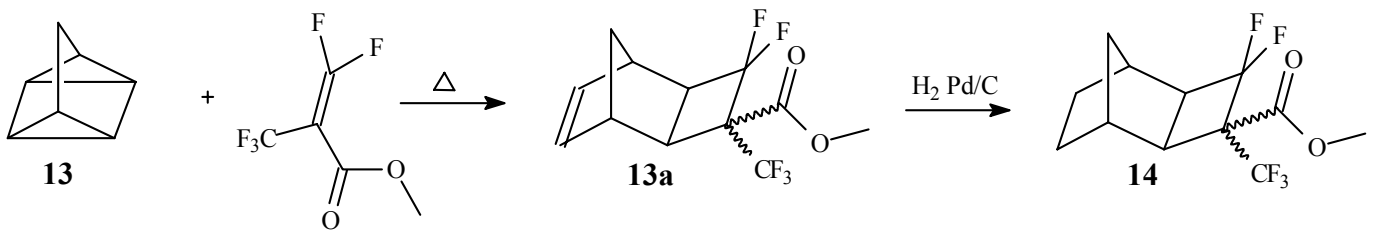

Figure 12. Synthesis of a fluorinated TCN compound
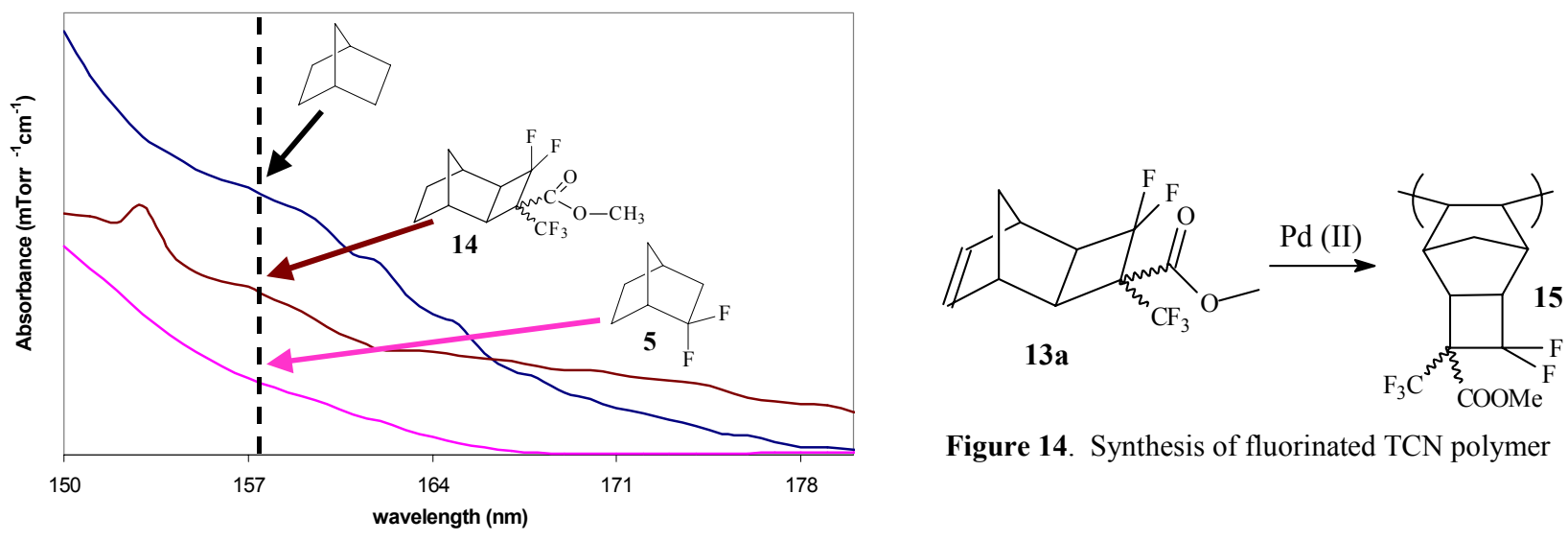

Figure 14. Synthesis of fluorinated TCN polymer

Figure 13. Absorbance of fluorinated TCN compound

\section{Section 3.4.2 Carbon Monoxide Copolymerization}

The use of carbon monoxide as a comonomer enabled the production of polymers from geminal $\mathrm{CF}_{3}$-substituted monomers that could otherwise not be polymerized by vinyl addition. Carbon monoxide polymerization yields two types of polymer backbones, a ketal form and a ketone form, depending on the type of catalyst, solvent and reaction temperature (Figure 15). ${ }^{10}$ The ketone form can be reduced or fluorinated by the appropriate reagents. ${ }^{11}$ These polymers will not be discussed in this paper. The current discussion will involve the spiral ketal form of the polymer. 


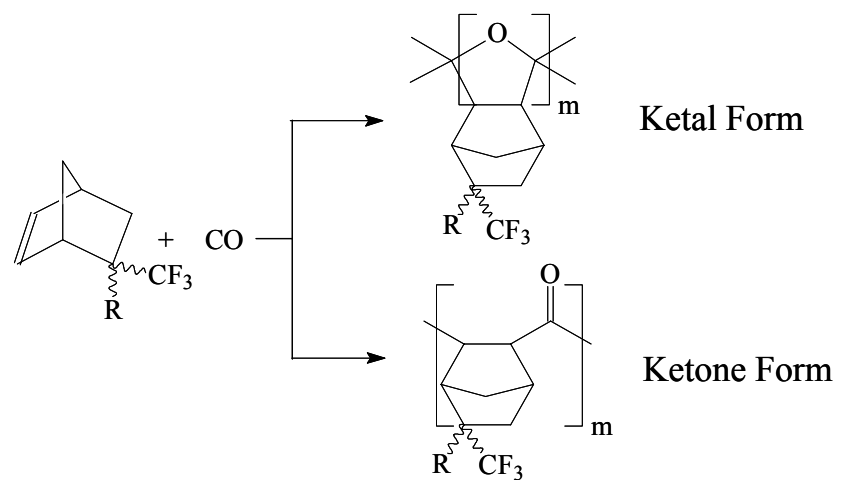

Figure 15. Products of carbon monoxide polymerization

We prepared two model ketal polymers $(\mathbf{1 6}, \mathbf{1 7})$ for absorbance measurements. Figure 16 shows the absorbance spectra of the models. Polymer 16, has an absorbance of $2.5 \mu^{-1}$ while the acetate-substituted polymer 17 has an absorbance of 3.1 $\mu \mathrm{m}^{-1}$. These materials are approximately one thousand times less absorbing at $157 \mathrm{~nm}$ than commercially available $248 \mathrm{~nm}$ resists. The main reason for studying these ketal polymers was to find a way to incorporate our geminal substituted norbornene monomers into a resist material. Therefore, monomer 18 was copolymerized with carbon monoxide using Drent's catalyst (Figure 17). The absorbance of the resulting polymer (19) is $4.0 \mu \mathrm{m}^{-1}$. Hydrolysis of the acetate provided polymer 20 with an absorbance of $2.7 \mu^{-1}$. Polymer 20a was also made successfully from the corresponding monomer (11). Clearly, it is possible to incorporate the geminal substituted norbornene monomers into a polymeric structure by an alternating copolymerization with carbon monoxide. All the carbon monoxide copolymers have low molecular weights (Mw $=1000-2500)$. The imaging characteristics of one of these copolymers will be discussed in a later section.
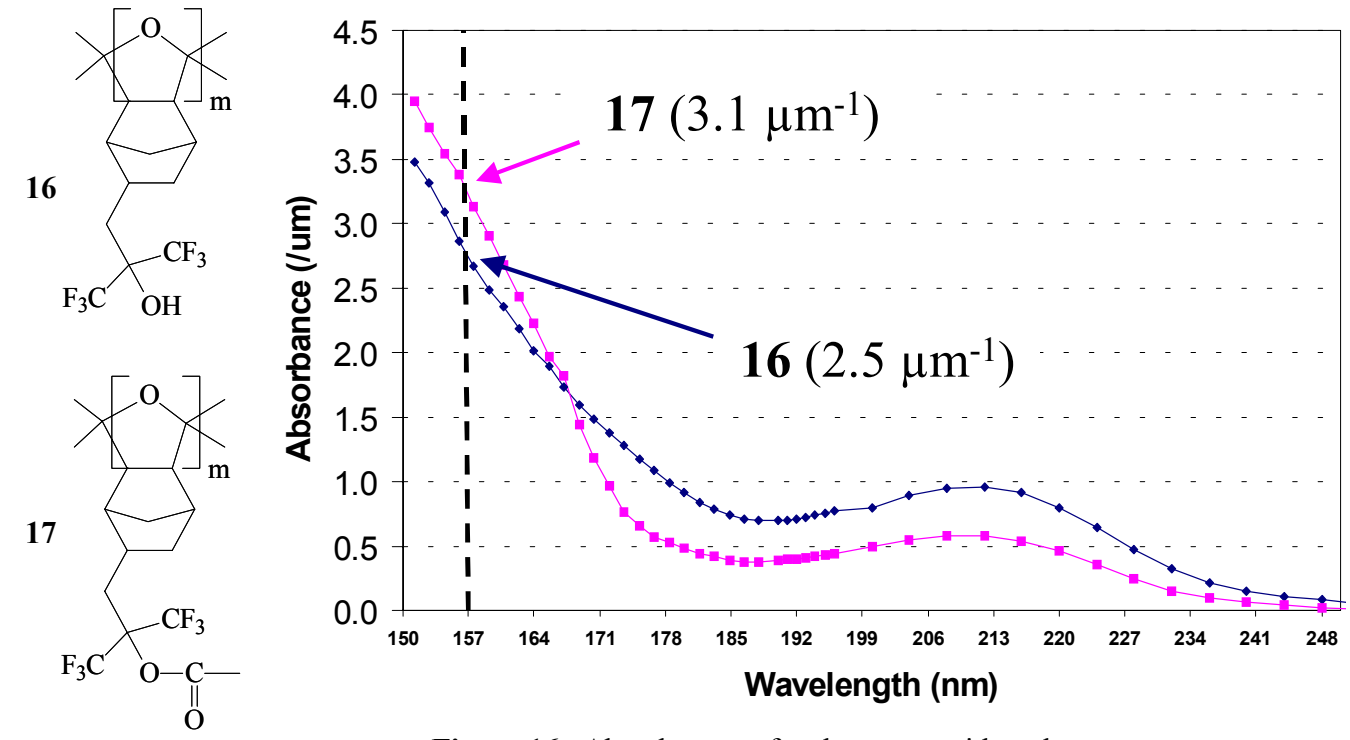

Figure 16. Absorbances of carbon monoxide polymers
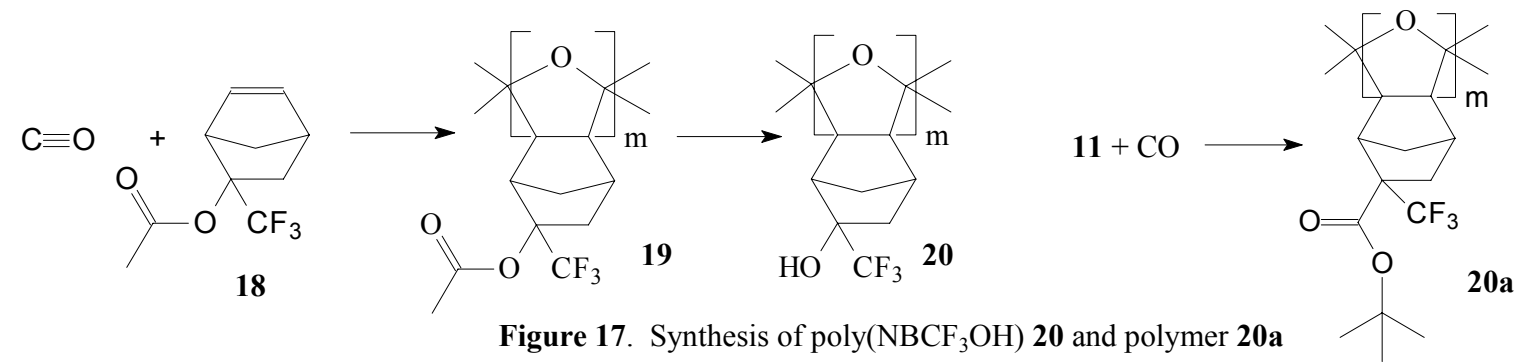

Figure 17. Synthesis of poly $\left(\mathrm{NBCF}_{3} \mathrm{OH}\right) 20$ and polymer 20a 


\section{Section 3.4.3 Radically-Initiated Copolymerization}

Preliminary work in the area of free-radical initiated polymerization has focused on the use of tetrafluoroethylene (TFE) as a comonomer. We are continuing the search for a transparent monomer that will undergo free-radical copolymerization with our norbornene monomers, analogous to the way maleic anhydride or sulfur dioxide undergoes radical polymerization with norbornene. A variety of comonomers are being auditioned in this study. A model polymer, poly(NB-co-TFE) (21), was made and its absorbance was found to be $1.1 \mu^{-1}$ (Figure 18). A functional derivative, poly(NBTBE-co-TFE) (22), has also been made, and its characterization is now in progress.
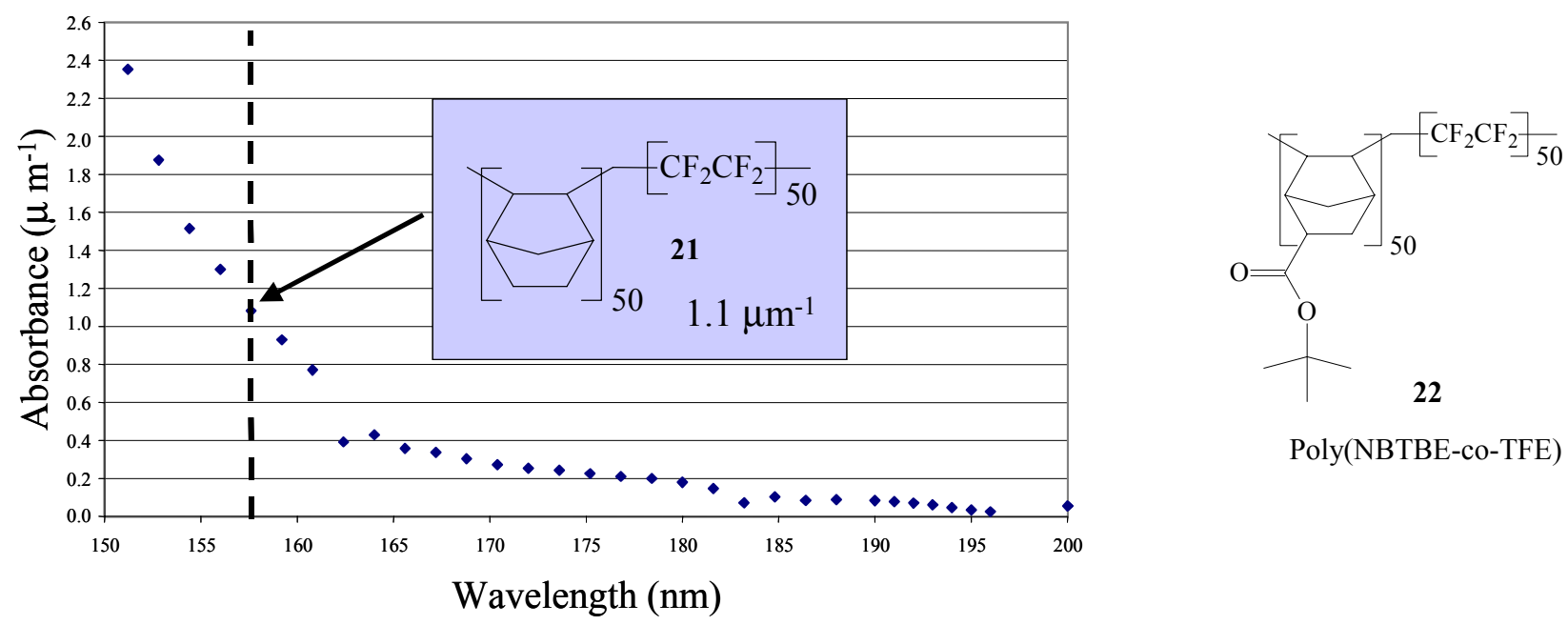

Poly(NBTBE-co-TFE)

Figure 18. Absorbance of poly(NB-co-TFE) 21

\section{Section 3.5 Lithographic Evaluation}

The functional characteristics of some of the polymers described in the paper have been assessed in preliminary imaging experiments.

\section{Section 3.5.1 Imaging of Carbon Monoxide Copolymers}

Preliminary $157 \mathrm{~nm}$ imaging studies were done on the carbon monoxide norbornene terpolymer 30. The resist formulation and imaging conditions are shown in Figure 20. This polymer showed good lithographic processing properties; the polymer coated well, wetted well during development, did not leave residues or swell after development, showed promising resolutions, and has high contrast. However, the surface of the developed resist was very rough. The roughness is presumed to be due to the polymer's poor mechanical properties, which in turn may be due to the low molecular weight and rod-like character of the polymer.
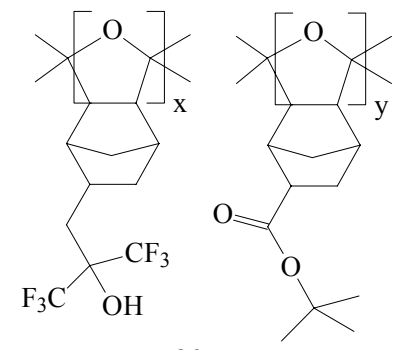

$$
\begin{aligned}
& \mathrm{Mw}=1200 \\
& \mathrm{x}=70, \mathrm{y}=30
\end{aligned}
$$


Formulation: $93.95 \%$ Polymer 30, 6\% TPS-Nf, 0.05\% TBAH in PGMEA

Conditions: $157 \mathrm{~nm}$ exposure (0.6NA- $\left.0.7 \sigma, 2 \mathrm{~mJ} / \mathrm{cm}^{2}\right)$, binary mask, $180 \mathrm{~nm}$ thick resist on $70 \mathrm{~nm}$ anti-reflective layer (DUV30), $130^{\circ} \mathrm{C}-60 \mathrm{~s}$ PAB, $130^{\circ} \mathrm{C}-90 \mathrm{~s}$ PEB, 20 sec $0.26 \mathrm{~N}$ TMAH development
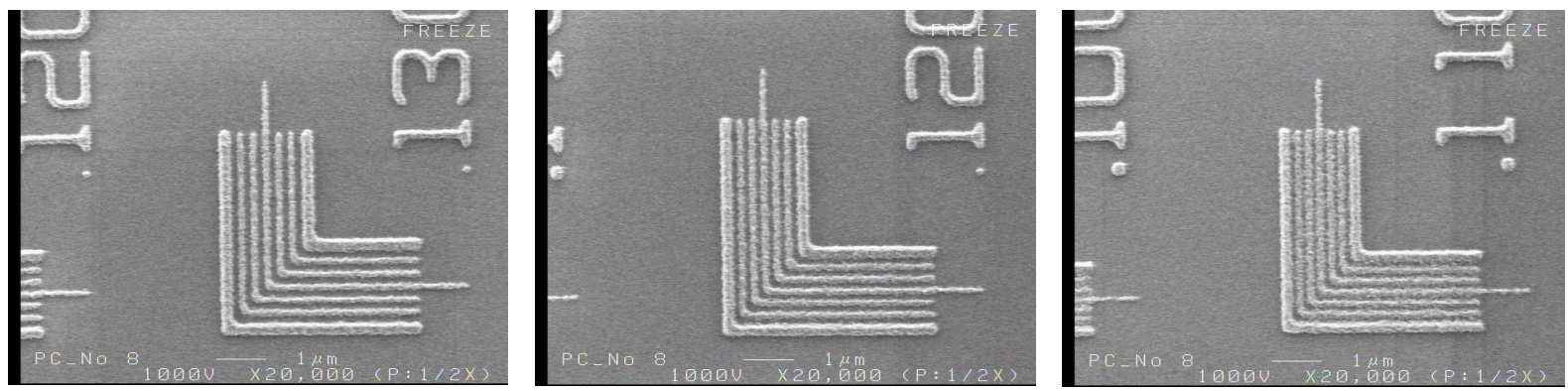

Figure 20. SEMs of carbon monoxide norbornene terpolymer 30

\section{Section 3.5.2 Dissolution Inhibitor (DI) Studies}

Efficient imaging of addition polymers is reported to require dissolution inhibitors. ${ }^{12}$ We have therefore surveyed a variety of compounds that may be used as dissolution inhibitors for $157 \mathrm{~nm}$ lithography. Since the carbon monoxide copolymers (20a, 23) typically have very low molecular weights, they were tested as dissolution inhibitors. Other compounds tested include 1,4-di(t-butoxy)tetrafluorobenzene (24), because it is sufficiently transparent at $157 \mathrm{~nm}$, and related monomeric species. The compounds were tested in polymer $25(\mathrm{Mw}=40,000)$, which was prepared by palladium(II) catalyzed vinyl addition polymerization.

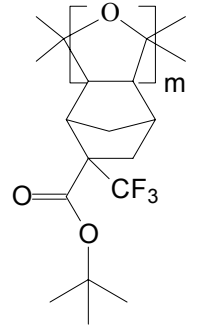

20a

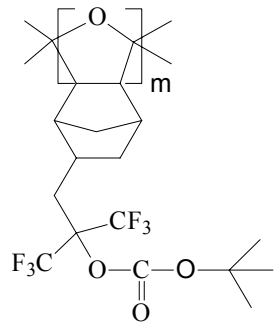

23

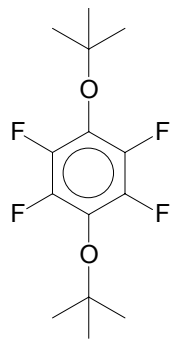

24

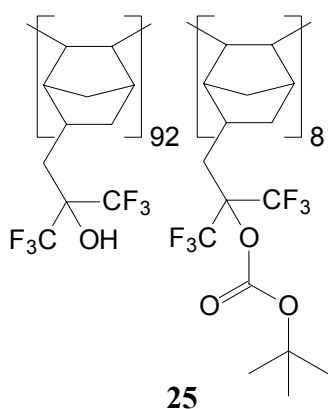

25

Solutions of $\mathbf{2 5}$ and each of the dissolution inhibitors were made in the appropriate solvent ( $20 \mathrm{wt} \%)$. The films were baked at $110^{\circ} \mathrm{C}$ for $90 \mathrm{sec}$ and thickness measurements were made before and after $120 \mathrm{sec}$ development time. The carbon monoxide copolymer blends gave clear, glassy films appear to be phase compatible with $\mathbf{2 5}$ over the entire concentration range studies. The dissolution rate was plotted against dissolution inhibitor loading, as shown in Figure 19.

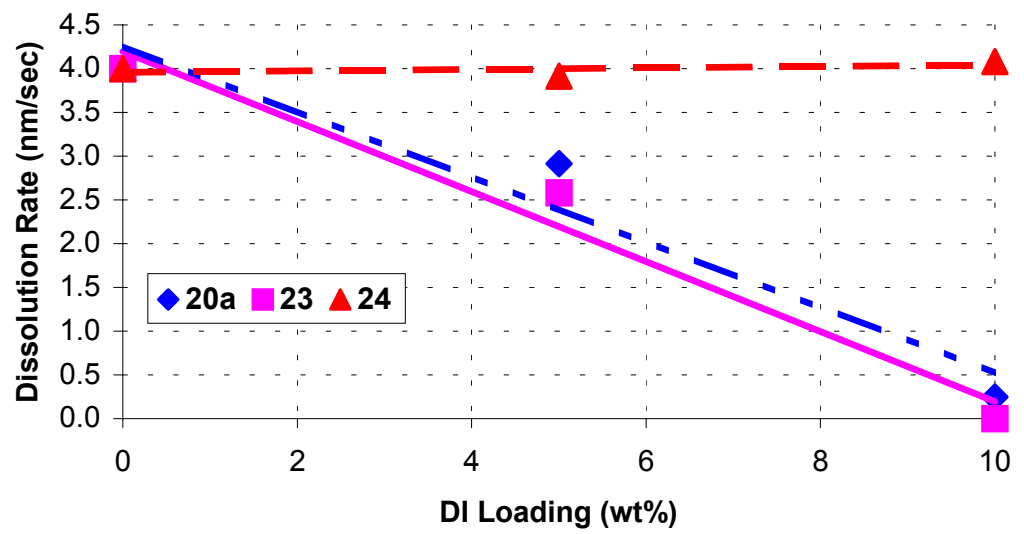

Figure 19. Dissolution rate of polymer 25 vs. DI Loading 
Compound 24 did not inhibit the dissolution of the base polymer at all, even at a high loading. Both of the carbon monoxide copolymers completely inhibit the dissolution of the base polymer at only $10 \mathrm{wt} \%$ loading. These two polymers are therefore both promising candidates for use as dissolution inhibitors for $157 \mathrm{~nm}$ lithography. We have also explored the use of other dissolution inhibitors. The structures of some of these compounds are shown below. The protecting group, R, may be $t$-BOC or $t$-butyl. These compounds do not have to be para-substituted, but can also be ortho- or meta-substituted.

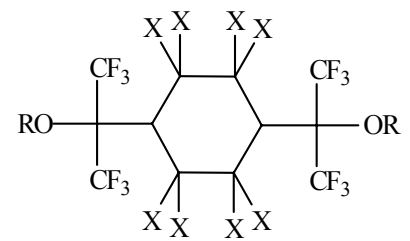

$\mathrm{X}=\mathrm{H}$ or $\mathrm{F}$

26<smiles>[R]Oc1c(F)c(F)c(F)c(F)c1F</smiles>

27

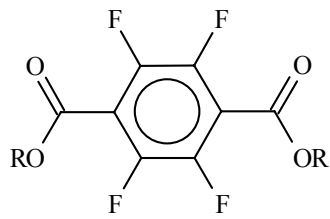

28

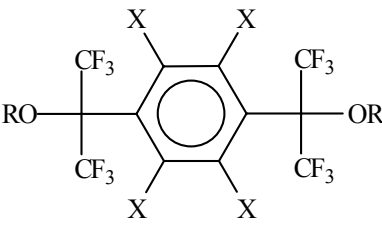

$\mathrm{X}=\mathrm{H}$ or $\mathrm{F}$

29

\section{Section 3.5.3 Imaging of Vinyl Addition Copolymers}

We are currently optimizing the formulations and imaging conditions required to print sub-100 $\mathrm{nm}$ features. This is a tremendous task since it is necessary to explore a variable space that includes photoacid generator, base, dissolution inhibitor, base polymer, exposure, bake, and development. Figure 21 shows some of the images we are able to print at this point in the program.

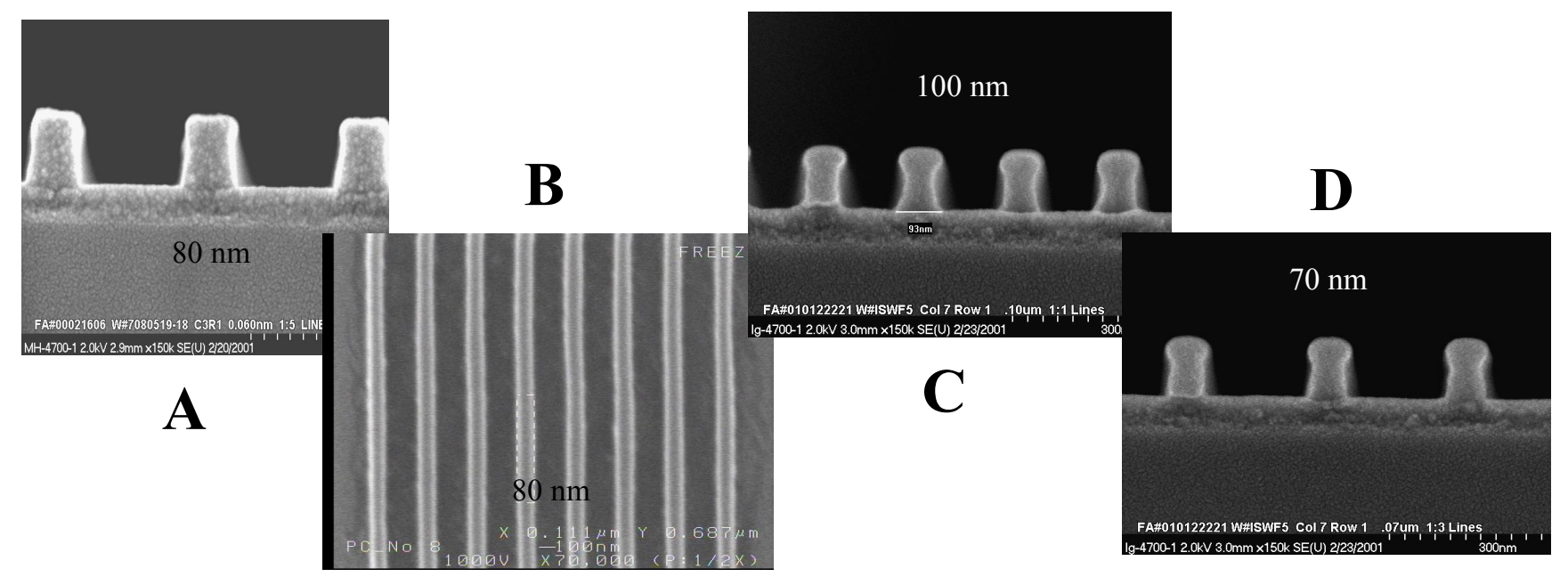

A, B: $50 / 50$ blend of $80 / 20$ vinyl addition copolymer $\mathbf{1 0}$ with CO copolymers 20a (DI)

C, D: $70 / 30$ blend of 70/30 vinyl addition copolymer $\mathbf{1 0}$ with CO copolymers 20a (DI)

Conditions: $157 \mathrm{~nm}$ exposure $\left(0.6 \mathrm{NA}-0.3 \sigma, 50 \mathrm{~mJ} / \mathrm{cm}^{2}\right)$, phase shift mask, $148 \mathrm{~nm}$ thick resist on $70 \mathrm{~nm}$ anti-reflective layer (DUV30), $140^{\circ} \mathrm{C}-60 \mathrm{~s} \mathrm{PAB}, 130^{\circ} \mathrm{C}-90 \mathrm{~s}$ PEB, 20 sec $0.26 \mathrm{~N}$ TMAH development.

Figure 21. SEMs of vinyl addition copolymers exposed at $157 \mathrm{~nm}$

\section{CONCLUSION}

We have shown that $\alpha$-trifluoromethyl carboxylic acid groups can serve as an acidic module in our "modular" design of 157 $\mathrm{nm}$ resist materials. These groups, along with the hexafluoroispropanol groups, are relatively transparent at the imaging wavelength. $t$-Butyl esters and $t$-BOC groups are acceptable protecting groups, even though the carbonyl contribution increases the absorbance. The carbonate group is surprisingly transparent. As our etch barrier, we have continued to use norbornene groups. The parent norbornane hydrocarbon is quite strongly absorbing at $157 \mathrm{~nm}$, but judicious fluorination of norbornene can significantly decrease this absorbance. Norbornene bearing geminal electron-withdrawing groups on the two carbon bridge are precursors to transparent polymers but these monomers resist direct polymerization by vinyl addition. Fortunately, several synthetic approaches were found to circumvent this problem. These include $\operatorname{Pd}($ II) catalyzed 
polymerization of TCN monomers, copolymerization of norbornene analogues with carbon monoxide, and free-radical copolymerization of norbornene analogues with TFE. The carbon monoxide copolymers have been shown to function as effective dissolution inhibitors for $157 \mathrm{~nm}$ lithography, and resist formulations based on addition polymers and these dissolution inhibitors have provided $70 \mathrm{~nm}$ images.

\section{ACKNOWLEDGEMENTS}

The authors gratefully acknowledge International SEMATECH for financial support of this work. Dr. Ralph Dammel from AZ Clariant and Central Glass Co. are also acknowledged for their generous donation of key monomers. We are indebted to JSR Corporation for support of Mr. Chiba, Shipley Co. for support of Dr. Yamada and ITRI for support of Dr. Vincent Jean. We are also indebted to Danny Miller and DC Owe-Yang from SEMATECH for their imaging work. Matthew Pinnow, Brian Osborn, and Colin Brodsky are gratefully acknowledged for their contributions to monomer synthesis and VUV measurements. Dr. Scott MacDonald is thanked for his helpful insights. Finally, we acknowledge the help of our undergraduates Anthony Vander Heyden and Jennifer Wunderlich.

\section{REFERENCES}

1. (a) Bloomstein, T. M.; Horn, M. W.; Rothschild, M.; Kunz, R. R.; Palmacci, S. T.; Goodman, R. B. J. Vac. Sci. Technol. $B$ 1997, 13(6), 2112. (b) Kunz, R. R.; Bloomstein, T. M.; Hardy, D. E.; Goodman, R. B.; Downs, D. K.; Curtin, J. E. Proc. SPIE 1999, 3678, 13-23.

2. Patterson, K.; Yamachika, M.; Hung, R.; Brodsky, C.; Yamada, S.; Somervell, M.; Osborn, B.; Hall, D.; Dukovic, G.; Byers, J.; Conley, W.; Willson, C. G. Proc. SPIE 2000, 3999, 365-374.

3. (a) Chiba, T.; Hung, R.; Yamada, S.; Trinque, B.; Yamachika, M.; Brodsky, C.; Patterson, K.; Vander Heyden, A.; Jamison, A.; Lin, S. H.; Somervell, M.; Byers, J.; Conley, W.; Willson, C. G. J. Photopolym. Sci. Technol. 2000, 13, 657-664. (b) Ito, H.; Schwalm, R. in Recent Advances in Anionic Polymerization, Hogen-Esch, T. E. and Smid, J., Ed., Elsevier, New York, 1987, 421-430.

4. Brodsky, C.; Byers, J.; Conley, W.; Hung, R.; Yamada, S.; Patterson, K.; Somervell, M.; Trinque, B.; Tran, H.V.; Cho, S.; Chiba, T.; Lin, S.H.; Jamieson, A.; Johnson, H.; Vander Heyden, T.; Willson, C.G. J. Vac. Sci. Technol. B 2000, 18(6), 3396-3401.

5. Tabushi, I.; Yamamura, K.; Yoshida, Z. J. Am. Chem. Soc. 1972, 94, 787.

6. (a) Risse et al. Makromol. Chem., Rapid Commun. 1991, 12, 255. (b) Okoroanyanwu, U. Ph.D. Dissertation, University of Texas at Austin, 1997. (b) Risse et al. Macromolecules, 1996, 29, 2755. (c) Goodall, B. L. et al. International patent, WO 9733198, 1997. (d) Klabunde et al. Organomet. 1985, 4, 571. (e) Rhodes et al. International patent, WO 9914256, 1999.

7. Drent, E. Eur. Pat. Appl. 351, 023, A1, 1990.

8. Ito, H. IBM J. Res. Dev. 1997, 41(1/2), 69-80.

9. Ito, H.; Giese, B.; Engelbrecht, R. Polym. Prepr. (Am. Chem. Soc., Div. Polym. Chem.) 1985, 26(1), 108-9.

10. Drent, E.; Budzelaar, P. H. M. Chem. Rev. 1996, 96, 663-681.

11. Larock, R. C. Comprehensive Organic Transformations: A Guide to Functional Group Preparations, 2nd Ed.; Wiley \& Sons: New York, 1999, 61-68, 689-690, 720-721, 1075-1089.

12. Ito, H.; Allen, R. D.; Opitz, J.; Wallow, T. I.; Truong, H. D.; Hofer, D. C.; Varanasi, P. R.; Jordhamo, G. M.; Jayaraman, S.; Vicari, R. Proc. SPIE 2000, 3999, 2-12.

13. Knunyants, I. L.; Zeifman, Yu. V.; Lushnikova, T. V.; Rokhlin, E. M.; Abduganiev, Yo. G.; Utebaev, U. J. Fluorine Chem.1975, 6, 227-240. 